\title{
Long-Term Immunogenicity of Live Oral Cholera Vaccine CVD 103-HgR in Adolescents Aged 12-17 Years in the United States
}

\author{
James M. McCarty, ${ }^{1 \star}$ David Cassie, ${ }^{2}$ Lisa Bedell, ${ }^{3}$ Michael D. Lock, ${ }^{3}$ and Sean Bennett ${ }^{3}$ \\ ${ }^{1}$ Stanford University School of Medicine, Stanford, California; ${ }^{2}$ Emergent BioSolutions Canada, Inc., Winnipeg, Canada; ${ }^{3}$ Emergent Travel Health, \\ Inc., Redwood City, California
}

\begin{abstract}
As part of a phase 4, randomized, double-blind, placebo-controlled trial to assess the immunogenicity and safety of PXVX0200 in children and adolescents aged 2-17 years, a subset of 73 adolescent subjects aged 12-17 years was followed for 2 years after vaccination and had blood collected for antibody assays on days 1, 11, 29, 91, 181, 365, 547, and 730. Endpoints included serum vibriocidal antibody (SVA) seroconversion, defined as a 4-fold or greater rise in antibody titer over baseline; geometric mean titers (GMTs); and geometric mean fold increase (GMFI) over baseline. Serum vibriocidal antibody seroconversion persisted in most subjects, with a rate of $64.5 \%$ noted at day 730 . Geometric mean titers and GMFI both peaked at day 11 and remained greater than baseline at all time points, including day 730 . Vaccination with PXVX0200 produces an immune response which persists for at least 2 years in adolescents aged 12-17 years.
\end{abstract}

Vibrio cholerae causes potentially life-threatening diarrhea and persists in much of the developing world. Cholera is endemic in 69 countries, with studies suggesting 1.3-4 million cases and $21,000-143,000$ deaths annually in endemic countries and 2,500 deaths annually in non-endemic countries. ${ }^{1}$ It also represents an ongoing risk for travelers to countries with endemic or epidemic cholera. ${ }^{2}$

Serum vibriocidal antibodies (SVAs) produced by natural or experimental $V$. cholerae infection correlate with protection against cholera. ${ }^{3,4}$ Experimental infection in adults resulted in protective immunity against rechallenge with both homologous and heterologous strains that lasted for at least 3 years. ${ }^{5}$ This led to the development of Center for Vaccine Development (CVD) 103-HgR (Emergent Travel Health, Redwood City, $C A)$, a live, attenuated strain of $V$. cholerae that does not produce active cholera toxin but induces a protective immune response. The vaccine was previously licensed in several countries under the names Mutachol, Orochol, and Orochol E but was discontinued for commercial reasons in 2001. CVD 103-HgR was acquired by PaxVax in 2009 and given the research name PXVX0200. Under the trade name VaxchoraTM (Emergent Travel Health, Redwood City, CA), it was licensed in the United States in 2016 for use in adults aged 18-64 years and in Europe in 2020 for use in individuals aged 6-64 years traveling to cholera-affected areas.

The efficacy of PXVX0200 was established in a phase three cholera challenge study in volunteers aged 18-45 years. In this trial, a single dose of PXVX0200 provided $90 \%$ and $80 \%$ protective efficacy against moderate-to-severe diarrhea following challenge at 10 days and 3 months, respectively, with $1 \times 10^{5}$ colony-forming units of wild-type $V$. cholerae O1 EI Tor Inaba strain $16961 .^{6}$ In the challenge study, SVA seroconversion was shown to be a strong correlate of protection. ${ }^{7}$ The safety and immunogenicity of PXVX0200 were further established in several immunologic bridging studies in subjects aged 2-64 years. ${ }^{8-11}$ However, the duration of immunity provided by PXVX0200 and SVA seroconversion, the correlate of protection, have not been studied beyond 6 months after

*Address correspondence to James M. McCarty, Stanford University School of Medicine, 291 Campus Dr., Stanford, CA 94305. E-mail: jmccart2@stanford.edu immunization. Here we report, for the first time, the long-term SVA response following vaccination with PXVX0200.

As part of a phase 4, randomized, double-blind, placebocontrolled trial to assess the immunogenicity and safety of PXVX0200 in children and adolescents aged 2-17 years, a subset of adolescent subjects aged 12-17 years was followed up for 2 years after vaccination. Study methods, including SVA methods, have been previously described..$^{10,12}$ In the long-term follow-up subset, vaccine recipients had blood collected for antibody assays on days $1,11,29,91,181,365,547$, and 730 , whereas placebo recipients were followed up through day 181 . The sub-study was performed at four sites in the United States from July 2017 to September 2019. Endpoints included SVA seroconversion, defined as a 4-fold or greater rise in antibody titer over baseline, geometric mean titers (GMTs) and geometric mean fold increase (GMFI) over baseline.

A total of 73 subjects enrolled in the long-term sub-study. The demographic and baseline characteristics of these subjects and the corresponding cohort from the main study up to day 181 were similar (Table 1). Serum vibriocidal antibody seroconversion persisted in most subjects, with a rate of $64.5 \%$ noted at day 730 (Table 2). Geometric mean titers and GMFI both peaked at day 11 and remained greater than baseline at all time points, including day 730 .

Cholera remains a threat to travelers of all ages to countries with endemic or epidemic disease and can be severe or fatal. Travelers at risk include those visiting friends and relatives, tourists, and relief workers. Those with blood group $O$ and medical conditions such as underlying heart, gastrointestinal, or renal disease are at increased risk for severe disease (cholera gravis). ${ }^{13,14} \mathrm{~A}$ single-dose vaccine which could rapidly provide long-term protection would be ideal.

Infection with naturally or experimentally acquired cholera induces serum vibriocidal antibodies, and these antibodies are associated with a reduced risk of cholera infection, with a stronger vibriocidal response resulting in greater protection. ${ }^{3,15}$ Whereas SVA response is a strong correlate of protection, the exact mechanism of protection is not completely understood, and SVA may be a proxy for a local intestinal immune response. ${ }^{16}$ One possibility is that vaccine- or infection-induced intestinal immunity is maintained by the presence of mucosal memory $B$ cells, which can rapidly differentiate into plasmablasts on cholera exposure. ${ }^{17}$

Experimental infection with $V$. cholerae mimics natural infection and induces protective immunity against rechallenge with both homologous and heterologous strains that lasts for 
TABLE 1

Long-term sub-study subject demography and baseline characteristics

\begin{tabular}{|c|c|c|}
\hline \multirow[b]{2}{*}{ Baseline characteristic } & \multicolumn{2}{|c|}{ Adolescents (12-17 years) } \\
\hline & Main study to day $181(N=163)$ & Long-term sub-study to day $730(N=73)$ \\
\hline \multicolumn{3}{|l|}{ Age (years) } \\
\hline Mean (SD) & $14.4(1.7)$ & $14.1(1.7)$ \\
\hline Median (min-max) & $14.0(12-17)$ & $14.0(12-17)$ \\
\hline \multicolumn{3}{|l|}{ Gender, $n(\%)$} \\
\hline Male & $88(54.0)$ & $38(52.1)$ \\
\hline Female & $75(46.0)$ & $35(47.9)$ \\
\hline \multicolumn{3}{|l|}{ Race, $n(\%)$} \\
\hline American Indian or Alaskan native & 0 & 0 \\
\hline Asian & $1(0.6)$ & $1(1.4)$ \\
\hline $\begin{array}{l}\text { Native Hawaiian or other Pacific } \\
\text { Islander }\end{array}$ & 0 & 0 \\
\hline Black or African American & $28(17.2)$ & $18(24.7)$ \\
\hline White & $121(74.2)$ & $44(60.3)$ \\
\hline Multiple & $13(8.0)$ & $10(13.7)$ \\
\hline Other & 0 & 0 \\
\hline \multicolumn{3}{|l|}{ Ethnicity, $n(\%)$} \\
\hline Hispanic or Latino & $18(11.0)$ & $10(13.7)$ \\
\hline Not Hispanic or Latino & $145(89.0)$ & $63(86.3)$ \\
\hline
\end{tabular}

at least 3 years. ${ }^{5}$ Previous studies with CVD 103-HgR demonstrated protection against rechallenge with heterologous biotypes and serotypes of $V$. cholerae up to 6 months after vaccination and evidence of persistent antibody response at 3.5 years. ${ }^{18-21}$ The duration of vaccine-derived protection produced by immunization with PXVX0200 is unknown and, given the persistence of serum vibriocidal antibodies for at least 2 years after immunization, it may be long term, although the degree of such protection is difficult to predict with confidence. In the cholera challenge trial, four of 68 PXVX0200 vaccinees at day 10 exhibited GMFI levels that were similar to those seen at days 365 and 730 in the current study. Half of those four subjects were protected from moderate or severe cholera when challenged later. ${ }^{6,7}$ Although this observation may suggest long-term protection, it is unclear how relevant GMFI levels measured 10 days after vaccination are to waning GMFI levels obtained a year or more after vaccination.

Ideally, prolonged protection could be definitively demonstrated by experimental challenge studies performed in volunteers 2-3 years after vaccination with PXVX0200. ${ }^{16}$ These studies are difficult to perform and are not without risk. The need for and timing of booster doses are also unknown at this time and should be studied. A rise in lipopolysaccharide (LPS) specific immunoglobulin A ( $\lg A)$ memory $B$ cells has also been shown to be significantly correlated with protection against cholera challenge following immunization with PXVX0200. ${ }^{22} \mathrm{lgA}$ and IgG LPS memory B-cell studies through day 730 are pending from the long-term immunogenicity substudy and may provide further evidence of enduring immunity.

In conclusion, vaccination with PXVX0200 produced an immune response which persisted for 2 years in adolescents aged 12-17 years. Because SVA seroconversion is a strong correlate of protection, PXVX0200 may provide protection against cholera infection for at least 2 years in individuals from developed countries when traveling to or visiting areas at risk for cholera. Long-term studies of cholera-specific memory $B$ cells are in progress and will further characterize the persistence of immunity.

TABLE 2

Long-term immunogenicity of PXVX0200 and placebo subjects in the IEP

\begin{tabular}{|c|c|c|c|c|c|c|}
\hline \multirow{2}{*}{$\begin{array}{l}\text { Visit }(\mathrm{PXVX0200,} \\
\text { placebo, } n)\end{array}$} & \multicolumn{2}{|c|}{$\begin{array}{c}\text { Serum vibriocidal antibody seroconversion rate } \\
\text { percentage, } 95 \% \mathrm{Cl}\end{array}$} & \multicolumn{2}{|c|}{ Geometric mean titer, $95 \% \mathrm{Cl}$} & \multicolumn{2}{|c|}{ Geometric mean fold increase, $95 \% \mathrm{Cl}$} \\
\hline & PXVX0200 & Placebo & PXVX0200 & Placebo & PXVX0200 & Placebo \\
\hline $\begin{array}{l}\text { Day } 1\left(n=72^{*}\right. \\
n=23)\end{array}$ & $\mathrm{n} / \mathrm{a}$ & $\mathrm{n} / \mathrm{a}$ & $32.4(26.2,40.0)$ & $43.8(27.5,69.6)$ & $\mathrm{n} / \mathrm{a}$ & $\mathrm{n} / \mathrm{a}$ \\
\hline $\begin{array}{l}\text { Day } 11(n=72, \\
n=23)\end{array}$ & $100 \%(94.9 \%, 100 \%)$ & $0(0.0 \%, 14.3 \%)$ & $9,035.4(6,745.3,12,103.0)$ & $41.2(26.1,65.1)$ & $279.2(212.4,366.9)$ & $0.9(0.8,1.1)$ \\
\hline $\begin{array}{c}\text { Day } 29(n=72, \\
n=23)\end{array}$ & $100 \%(94.9 \%, 100 \%)$ & $0(0.0 \%, 14.3 \%)$ & $2,791.7(2,176.4,3,580.9)$ & $42.5(27.1,66.7)$ & $86.3(67.6,110.1)$ & $1.0(0.8,1.2)$ \\
\hline $\begin{array}{l}\text { Day } 91(n=72, \\
n=23)\end{array}$ & $88.9 \%(79.6 \%, 94.3 \%)$ & $0(0.0 \%, 14.3 \%)$ & $391.7(293.9,522.1)$ & $42.5(28.7,62.9)$ & $12.1(9.3,15.8)$ & $1.0(0.7,1.3)$ \\
\hline $\begin{array}{l}\text { Day } 181(n=71, \\
n=21)\end{array}$ & $83.1 \%(72.7 \%, 90.1 \%)$ & $0(0.0 \%, 15.5 \%)$ & $223.0(166.5,298.6)$ & $38.7(25.8,58.0)$ & $6.9(5.4,8.9)$ & $0.9(0.8,1.1)$ \\
\hline $\begin{array}{l}\text { Day } 365(n=70, \\
\text { n/a) }\end{array}$ & $68.6 \%(57.0 \%, 78.2 \%)$ & - & $158.4(121.6,206.4)$ & - & $4.8(3.8,6.1)$ & - \\
\hline $\begin{array}{l}\text { Day } 547(n=67, \\
\text { n/a) }\end{array}$ & $73.1 \%(61.5 \%, 82.3 \%)$ & - & $175.6(134.1,229.9)$ & - & $5.2(4.2,6.6)$ & - \\
\hline $\begin{array}{l}\text { Day } 730(n=62, \\
\text { n/a) }\end{array}$ & $64.5 \%(52.1 \%, 75.3 \%)$ & - & $133.8(101.9,175.7)$ & - & $4.1(3.2,5.2)$ & - \\
\hline
\end{tabular}


Received December 11, 2020. Accepted for publication February 28, 2021.

Published online April 5, 2021.

Authors' addresses: James M. McCarty, Department of Pediatrics, Stanford University School of Medicine, Stanford, CA, E-mail: jmccart2@stanford.edu. David Cassie, Clinical Research, Emergent BioSolutions Canada, Inc., Winnipeg, Canada, E-mail: dcassie@ ebsi.com. Lis Bedell and Michael D. Lock, Department of Biostatistics, Emergent Travel Health, Inc., Redwood City, CA, E-mails: bedell@ ebsi.com and mike@lockstats.com. Sean Bennett, Clinical Development, Emergent Travel Health, Inc., Redwood City, CA, E-mail: sbennett22903@ gmail.com.

This is an open-access article distributed under the terms of the Creative Commons Attribution (CC-BY) License, which permits unrestricted use, distribution, and reproduction in any medium, provided the original author and source are credited.

\section{REFERENCES}

1. Ali M, Nelson AR, Lopez AL, Sack DA, 2015. Updated global burden of cholera in endemic countries. PLoS Negl Trop Dis 9: e0003832.

2. Mascarello M, Deiana M, Maurel C, Lucarelli C, Luzzi I, Luzzati R, 2013. Cholera with severe renal failure in an Italian tourist returning from Cuba, July 2013. Eurosurveillance 18: 20572.

3. Mosley WH, Ahmad S, Benenson AS, Ahmed A, 1968. The relationship of vibriocidal antibody titre to susceptibility to cholera in family contacts of cholera patients. Bull World Health Organ 38: 777-785.

4. Mosley WH, McCormack WM, Ahmed A, Chowdhury AK, Barui RK, 1969. Report of the 1966-1967 cholera vaccine field trial in rural east Pakistan. 2. Results of the serological surveys in the study population--the relationship of case rate to antibody titre and an estimate of the inapparent infection rate with Vibrio cholerae. Bull World Health Organ 40: 187-197.

5. Levine MM, Black RE, Clements ML, Cisneros L, Nalin DR, Young CR, 1981. Duration of infection-derived immunity to cholera. $J$ Infect Dis 143: 818-820.

6. Chen WH et al., 2016. Single-dose live oral cholera vaccine CVD $103-\mathrm{HgR}$ protects against human experimental infection with Vibrio cholerae O1 El Tor. Clin Infect Dis 62: 1329-1335.

7. Haney DJ, Lock MD, Simon JK, Harris J, Gurwith M, 2017. Antibody-based correlates of protection against cholera analysis of a challenge study in a cholera-Naïve population. Clin Vaccine Immunol 24: e00098-17.

8. McCarty JM, Lock MD, Hunt KM, Simon JK, Gurwith, 2018. Safety and immunogenicity of single-dose live oral cholera vaccine strain CVD 103-HgR in healthy adults age 18-45. Vaccine 36 : 833-840.

9. McCarty JM, Lock M, Bennett S, Hunt K, Simon J, Gurwith M, 2019. Age related immunogenicity and reactogenicity of live oral cholera vaccine CVD 103-HgR in a randomized, controlled clinical trial. Vaccine 37: 1389-1397.

10. McCarty JM, Gierman EC, Bedell L, Lock MD, Bennet S, 2020. Safety and immunogenicity of live oral cholera vaccine CVD $103-\mathrm{HgR}$ in children and adolescents aged 6-17 years. Am J Trop Med Hyg 102: 48-57.

11. McCarty JM, Cassie D, Bedell L, Lock MD, Bennet S, 2021. Safety and immunogenicity of live oral cholera vaccine CVD 103-HgR in children 2-5 years of age in the US. Am J Trop Med Hyg 104: 861-865.

12. Beneson AS, Saad A, Mosley WH, 1968. Serological studies in cholera. 2. The vibriocidal antibody response of cholera patients determined by a microtechnique. Bull World Health Organ 38: 277-285.

13. Levine MM, Nalin DR, Rennels MB, Hornick RB, Sotman S, Van Blerk G, Hughes TP, O'Donnell S, Barua D, 1979. Genetic susceptibility to cholera. Ann Hum Biol 6: 369-374.

14. Weil AA, Khan AI, Chowdhury F, Larocque RC, Faruque ASG, Ryan ET, Calderwood SB, Qadri F, Harris JB, 2009. Clinical outcomes in household contacts of patients with cholera in Bangladesh. Clin Infect Dis 49: 1473-1479.

15. Clements ML, Levine MM, Young CR, Black RE, Lim YL, RobinsBrowne RM, Craig JP, 1982. Magnitude, kinetics, and duration of vibriocidal antibody responses in North Americans after ingestion of Vibrio cholerae. J Inf Dis 145: 465-473.

16. Levine MM, Chen WH, Kaper JB, Lock M, Danzig L, Gurwith M, 2017. PaxVax CVD 103-HgR single-dose live oral cholera vaccine. Expert Rev Vaccin 16: 197-213.

17. Harris JB, 2018. Cholera: immunity and prospects in vaccine development. J Infect Dis 218 (Supp/ 3): S141-S146.

18. Levine M, Herrington D, Losonsky G, Tall B, Kaper J, Ketley J, Tacket CO, Cryz S, 1988. Safety, immunogenicity, and efficacy of recombinant live oral cholera vaccines, CVD 103 and CVD 103-HgR. Lancet 332: 467-470.

19. Tacket CO, Losonsky G, Nataro JP, Cryz SJ, Edelman R, Kaper JB, Levine M, 1992. Onset and duration of protective immunity in challenged volunteers after vaccination with live oral cholera vaccine CVD I03-HgR. J Infect Dis 166: 837-841.

20. Tacket $\mathrm{CO}$ et al., 1999. Randomized, double-blind, placebocontrolled, multicentered trial of the efficacy of a single dose of live oral cholera vaccine CVD 103-HgR in preventing cholera following challenge with Vibrio cholerae O1 El Tor Inaba three months after vaccination. Infect Immun 67: 6341-6345.

21. Kollaritsch H, Cryz S, Lang A, Herzog C, Que J, Wiedermann G, 2000. Local and systemic immune responses to combined Vibrio cholerae CVD103-HgR and Salmonella typhi Ty21a live oral vaccines after primary immunization and reimmunization. Vaccine 18: 3031-3039.

22. Haney DJ et al., 2018. Lipopolysaccharide-specific memory B cell responses to an attenuated live cholera vaccine are associated with protection against Vibrio cholerae infection. Vaccine 36 : 2768-2773. 